'Escuela de Kinesiología, Facultad de Salud, Universidad Santo Tomás. Chile.

2Pedagogía en Educación Física, Facultad de Educación, Universidad Autónoma de Chile. Chile. ${ }^{3}$ Departamento de Ciencias del Deporte y Acondicionamiento Físico. Universidad Católica de la Santísima Concepción. Concepción, Chile. ${ }^{4}$ Instituto de Anatomía, Histología y Patología. Facultad de Medicina, Universidad Austral de Chile. Valdivia, Chile.

Instituto de Farmacia, Facultad de Ciencias, Universidad Austral de Chile. Valdivia, Chile. ${ }^{6}$ Centro de Investigación en Educación y Desarrollo (CIEDE-UCSC), Departamento de Salud Pública, Facultad de Medicina, Universidad Católica de la Santísima Concepción. Concepción, Chile. ${ }^{7}$ Centro de Vida Saludable de la Universidad de Concepción y Departamento de Bioquímica Clínica e Inmunología, Facultad de Farmacia, Universidad de Concepción. Concepción, Chile. ${ }^{8}$ BHF Glasgow Cardiovascular Research Centre, Institute of Cardiovascular and Medical Sciences, University of Glasgow, Glasgow, United Kingdom.

Institute of Health and Wellbeing, University of Glasgow. Glasgow, United Kingdom. ${ }^{10}$ Escuela de Pedagogía en Educación Física, Depto. Cs de la Educación, Grupo Calidad de vida en diferentes poblaciones, Facultad de Educación y Humanidades, Universidad del Biobío. Chile.

"Laboratorio de Rendimiento Humano, Grupo de Estudio en Educación, Actividad Física y Salud (GEEAFyS), Universidad Católica del Maule. Talca, Chile. ${ }^{12}$ Centro de Investigación en Fisiología del Ejercicio (CIFE), Universidad Mayor. Chile. aKinesiólogo, MSc en Ciencias de la Actividad Física y Salud.

bKinesiólogo, Doctor en Neurociencias. 'Profesor de Educación Física, Doctor en Actividad Física, Educación Física y Deporte. dProfesor de Biología y Química. MSc. Neurociencias y Salud Mental.

eBioquímico, MSc Nutrición y Dietética. ${ }^{\mathrm{f}}$ Nutricionista, MSc. Salud Pública basada en la evidencia.

9Bioquímico, Doctor en Ciencias Biológicas, mención Biología molecular.

hKinesiólogo, MSc Sports and Exercise Science \& Medicine.

'Nutricionista, MSc. Nutrición Humana.

jNutricionista, MSc. en Nutrición y Alimentos mención Promoción de la Salud.

kProfesor de Educación Física, Doctor en Innovación didáctica y Formación de Profesorado.

'Profesor de Educación Física, Doctor Ciencias Cardiovasculares y Biomédicas.

Trabajo no recibió financiamiento. Los autores declaran no tener conflictos de interés.

Recibido el 28 de marzo de 2020, aceptado el 10 de noviembre de 2020

Correspondencia a:

Dr. Carlos Celis-Morales

BHF Glasgow Cardiovascular Research Centre, Institute of Cardiovascular and Medical Sciences. College of Medical, Veterinary and Life Sciences. University of Glasgow, Glasgow G12 8TA. United Kingdom. Carlos.Celis@glasgow.ac.uk

\section{Prevalencia de debilidad muscular en personas mayores chilenas: resultados de la Encuesta Nacional de Salud 2016-2017}

\author{
YENY CONCHA-CISTERNAS ${ }^{1,2, a}$, \\ IGOR CIGARROA $^{1, \mathrm{~b}}$, \\ CARLOS MATUS-CASTILLO ${ }^{3, \mathrm{c}}$, \\ ALEX GARRIDO-MÉNDEZ ${ }^{3, \mathrm{c}}$, \\ ANA MARÍA LEIVA-ORDOÑEZ ${ }^{4, \mathrm{~d}}$, \\ MARÍA ADELA MARTÍNEZ-SANGUINETTI ${ }^{5, \mathrm{e}}$, \\ CLAUDIA TRONCOSO-PANTOJA ${ }^{6, \mathrm{f}}$, NATALIA ULLOA ${ }^{7, \mathrm{~g}}$, \\ MARÍA FERNANDA GABLER ${ }^{8, \mathrm{~h}}$, \\ FANNY PETERMANN-ROCHA ${ }^{8,9, \mathrm{i}}$, \\ SOLANGE PARRA-SOTO ${ }^{8,9, \mathrm{j}}$, XIMENA DÍAZ ${ }^{10, \mathrm{k}}$, \\ CARLOS CELIS-MORALES ${ }^{8,9,11,12,1}$
} (en representación de todos los integrantes del Consorcio ELHOC, Epidemiology of Lifestyle and Health Outcomes in Chile)

\section{Prevalence of low hand grip strength in Chilean older adults. Findings from the national health survey 2016-2017}

Background: Handgrip strength is an indicator of frailty in older people. Aim: To determine the prevalence of low handgrip strength in older Chilean adults. Material and Methods: A cross-sectional analysis of 244 individuals aged 60 years or more, participating in the 2016-2017 Chilean National Health Survey, was carried out. Handgrip strength was evaluated by a hand dynamometer and low grip strength was determined as a grip strength $\leq 15 \mathrm{~kg}$ and $\leq 27 \mathrm{~kg}$ for women and men, respectively. Results: Twenty nine percent of participants had low grip strength. The average grip strength among 60-year-old men and women was 34.7 and $22.1 \mathrm{~kg}$, respectively. These figures decreased to 28.8 $\mathrm{kg}$ and $17.2 \mathrm{~kg}$ among 90-year-old men and women, respectively. The prevalence of low grip strength in men and women aged 60 years was 18\%. In 90-year-old men and women, these figures increased to $79 \%$ and $56.3 \%$, respectively. Conclusions: The prevalence of low grip strength increased substantially with age.

(Rev Med Chile 2020; 148: 1598-1605)

Key words: Chile; Frail Elderly; Hand Strength; Frailty. 
$\mathrm{E}$ lenvejecimiento es un proceso que provoca cambios anatomofisiológicos sobre los sistemas corporales ${ }^{1}$. Dentro de estos cambios, los que afectan al sistema músculo-esquelético se han asociado con importantes modificaciones sobre habilidades motoras básicas como la marcha, además de relacionarse con deterioro funcional y pérdida de autonomía ${ }^{2,3}$.

A partir de los 20 años la masa muscular disminuye en promedio 0,4 a $0,8 \mathrm{~kg}$ por década, siendo mayor esta pérdida en hombres $(1 \%)$ que en mujeres $(0,5 \%)^{4}$. Existe también disminución en el área de sección transversal muscular y pérdida en el número y tamaño de las fibras musculares ${ }^{5}$, eventos que desencadenan la aparición de debilidad muscular, sarcopenia y fragilidad ${ }^{6}$.

Del mismo modo, se ha reportado que conforme avanza la edad aumenta el número de citoquinas antiinflamatorias y proinflamatorias como las interleuquinas (IL) IL-1, IL-6, IL-15 y TNF- $\alpha$ (factor de necrosis tumoral- $\alpha$ ), las cuales pueden modular la homeostasis entre la síntesis de proteínas y la degradación muscular. Esta pérdida del equilibrio provocaría pérdida de masa muscular, y con ello debilidad muscular ${ }^{7}$.

Si bien la debilidad muscular en personas mayores es generalizada, estudios señalan que afecta principalmente a la musculatura apendicular, reportándose que entre $16 \%$ a $18 \%$ de las mujeres, y entre $8 \%$ a $10 \%$ de los hombres mayores de 65 años no pueden levantar ni tomar 4,5 kilogramos de peso ${ }^{9}$. Además, presentan problemas para agacharse o arrodillarse, tareas esenciales que dependen de la indemnidad de la fuerza muscular del tren superior e inferior?.

Una forma de cuantificar la debilidad muscular es a través de la fuerza de prensión manual, la cual es calificada como una prueba de fácil ejecución y bajo costo ${ }^{10}$. De acuerdo a estudios previos, una baja fuerza de prensión manual se relaciona con eventos adversos de salud ${ }^{11-13}$, además de asociarse inversamente con mortalidad por todas las causas $^{11,12}$. Así mismo, se ha reportado asociación positiva entre la prueba de prensión manual con cáncer de pulmón en países desarrollados, con enfermedad pulmonar obstructiva crónica, y con pérdida de la fuerza global en personas mayores, pudiendo ser considerada, un predictor funcional para ejecutar actividades de la vida diaria ${ }^{11,14}$.

En Chile, diversas investigaciones han reportado valores de referencia para la fuerza de prensión manual en todas las edades ${ }^{15-17}$. Gomez-Campos y cols. establecieron valores de fuerza de prensión manual en población entre 6 y 17 años ${ }^{15}$, mientras que Romero y cols. establecieron tablas de normalidad de fuerza de prensión para personas entre 20 y 70 años $^{16}$. Por otra parte, Lera y cols. desarrollaron valores normativos en personas mayores de 60 años $^{17}$. Establecer valores de referencia es importante ya que, permite contar con puntos de riesgo de limitación funcional los cuales podrían estar asociados a un mayor riesgo de mortalidad prematura, ayudando a la identificación de pacientes con debilidad muscular y por ende, un mayor riesgo de mortalidad en la práctica clínica. Del mismo modo, determinar la prevalencia de debilidad muscular podría ser de interés para lograr estimar la cantidad de personas mayores que actualmente presentan debilidad muscular en Chile, y para ayudar en la orientación de evaluaciones clínicas y los objetivos de trabajo centrados en la mejora de capacidades funcionales en personas mayores.

Es por lo expuesto, que el objetivo de este trabajo fue determinar la prevalencia de debilidad muscular mediante la fuerza de prensión manual en personas chilenas mayores de 60 años.

\section{Materiales y Métodos}

\section{Diseño del estudio}

Este estudio utilizó datos de la Encuesta Nacional de Salud 2016-2017 (ENS 2016-2017). Dicha encuesta correspondió a un estudio poblacional de tipo transversal, probabilístico estratificado geográficamente, multietápico y de conglomerados de 6.233 personas chilenas mayores de 15 años, provenientes de zonas urbanas y rurales de 15 regiones del país. Sin embargo, el presente estudio solo incluyó a una submuestra de 243 de un total de 293 personas mayores de la región Metropolitana ( $\geq 60$ años). Las personas excluidas $(n=50)$ correspondieron a personas que no pudieron realizar el test de prensión debido a la existencia de patologías que les impedían realizar dicha prueba. Para ponderar la muestra a población nacional, se aplicaron los factores de expansión sugeridos por la ENS 2016-2017 quedando una muestra expandida de 1.103 .515 personas mayores en Chile.

El protocolo de estudio fue aprobado por el Comité de Ética de la Facultad de Medicina de la 
Pontificia Universidad Católica de Chile y todos los participantes firmaron un consentimiento informado ${ }^{18}$.

\section{Prevalencia de debilidad muscular y fuerza de prensión manual}

La fuerza muscular fue estimada mediante la prueba de prensión manual (Grip Strength Test) utilizando un dinamómetro hidráulico marca JAMAR ${ }^{\circledR}$ Sammons Preston Inc. previamente calibrado. Esta evaluación se llevó a cabo con el sujeto sentado en una silla con respaldo, hombros aducidos, codo flectado en $90^{\circ}$, antebrazo y muñeca en posición neutra. El brazo evaluado no tenía apoyo en ninguna superficie y el dinamómetro se utilizó en posición vertical. Se solicitó a los participantes realizar una fuerza de prensión máxima con su mano dominante durante 3 segundos, con reposo de 1 minuto entre cada repetición, realizando dos intentos ${ }^{16,18}$. Se utilizó el valor más alto de prensión de las repeticiones. El protocolo de evaluación se realizó en el hogar del participante y fue ejecutado por profesionales de salud capacitados. Este protocolo es descrito en los manuales entregados en la ENS 2016-2017

El valor registrado se expresó en $\mathrm{kg}$ de fuerza en una escala de $0-90 \mathrm{~kg}$. Se consideró con debilidad muscular a las mujeres que obtuvieron $\leq 15 \mathrm{~kg}$ de fuerza en la prueba de prensión manual y $\leq 27 \mathrm{~kg}$ en varones. Estos valores de corte fueron determinados a partir de estudios nacionales ${ }^{17}, \mathrm{y}$ concuerdan con los sugeridos por estudios internacionales ${ }^{11}$ para cuyos valores se ha observado un mayor incremento en el riesgo de incidencia de enfermedades y mortalidad específica. Además, el Grupo de Trabajo Europeo sobre Sarcopenia en Personas Mayores (EWGSOP2) del año 2019 estableció el mismo punto de corte para varones mayores ${ }^{19}$.

\section{Variables sociodemográficas, antropométricas y de estilo de vida}

Las variables sociodemográficas edad, grupo etario, sexo (hombre o mujer), zona de residencia (rural o urbano), nivel educacional (básica $<8$ años, media 8 a 12 años o educación superior $>12$ años), estilos de vida y autopercepción de salud y bienestar personal, se obtuvieron mediante la aplicación de cuestionarios de la ENS 2016-2017 ${ }^{18}$. El estado nutricional fue clasificado en base al índice de masa corporal (IMC), el cual se obtiene dividiendo el peso corporal por la altura bípeda al cuadrado (peso/altura $\left.{ }^{2}\right)^{20}$. Se consideraron puntos de corte de valoración específica para el adulto mayor (bajo peso: $<22,9 \mathrm{~kg} / \mathrm{m}^{2}$; normo peso: $23,0-27,9 \mathrm{~kg} / \mathrm{m}^{2}$; sobrepeso: $28,0-31,9 \mathrm{~kg} / \mathrm{m}^{2}$ y obesidad: $\left.\geq 32,0 \mathrm{~kg} / \mathrm{m}^{2}\right)^{20}$. La obesidad central fue definida como un perímetro de cintura (PC) $\geq 88 \mathrm{~cm}$ para mujeres $\mathrm{y} \geq 102 \mathrm{~cm}$ para hombres ${ }^{21}$.

El nivel de actividad física y sus intensidades fueron determinados mediante la aplicación del cuestionario "Global Physical Activity Questionnai$r e$ ” (GPAQ v2) el cual ha sido validado internacionalmente y en población latina ${ }^{22,23}$. La prevalencia de inactividad física fue determinada con los puntos de corte sugeridos por la Organización Mundial de la Salud y los niveles de sedentarismo fueron determinados mediante auto-reporte ${ }^{23,24}$.

\section{Análisis estadístico}

Se utilizó el software STATA MP15 y los comandos de muestras complejas para encuestas poblacionales para realizar los análisis estadísticos. Los datos de la población estudiada son presentados como promedios para variables continuas o como proporción para variables categóricas con su respectivo intervalo de confianza (95\% IC).

Para determinar la asociación entre edad y fuerza de prensión, se utilizó análisis de regresión. Los valores de fuerza, expresados en kg junto con sus respectivos IC según grupo etario, fueron estimados con el comando "margins" para muestras expandidas. La prevalencia de debilidad muscular según edad fue estimada mediante análisis de regresión logística para muestras expandidas y sus resultados son presentados como prevalencia junto a 95\% IC.

\section{Resultados}

Las características de la población con y sin debilidad muscular se muestran en la Tabla 1 . Se evidenció una prevalencia total de debilidad muscular del 29,1\%. Los participantes con debilidad muscular fueron principalmente hombres $(31,3 \%)$ entre 76 y 80 años, con menos de 8 años de escolaridad. En cuanto a las características antropométricas y nutricionales, los participantes con debilidad muscular presentaron menor peso corporal, IMC y PC, no obstante, según estado nutricional, las personas con debilidad muscular principalmente presentaron bajo peso u obesidad. Este grupo 
Tabla 1. Características de la población según debilidad muscular

\begin{tabular}{|c|c|c|}
\hline & Normal & Debilidad muscular \\
\hline Número encuestados* & 171 & 73 \\
\hline Número expandido a población nacional* & 782,710 & 320,805 \\
\hline Prevalencia expandida** & $70,9(6,4 ; 78,1)$ & $29,1(21,8 ; 37,5)$ \\
\hline Edad, (años) & $68,5(66,9 ; 70,3)$ & $72,5(69,3 ; 75,3)$ \\
\hline \multicolumn{3}{|l|}{ Sexo, (\%) } \\
\hline Mujeres & $73,1(62,7 ; 81,3)$ & $26,9(18,6 ; 37,2)$ \\
\hline Hombres & $68,6(54,5 ; 79,9)$ & $31,4(20,0 ; 45,4)$ \\
\hline $\begin{array}{l}\text { Grupos etarios, }(\%) \\
60-65 \text { años } \\
66-70 \text { años } \\
71-75 \text { años } \\
76-80 \text { años } \\
80 \text { y más }\end{array}$ & $\begin{array}{l}79,2(65,0 ; 88,6) \\
76,3(58,8 ; 87,9) \\
66,0(46,6 ; 81,1) \\
45,9(25,1 ; 68,2) \\
63,3(37,6,83,2)\end{array}$ & $\begin{array}{l}20,7(11,3 ; 34,9) \\
23,6(12,0 ; 41,1) \\
33,9(18,8 ; 53,3) \\
54,0(31,7 ; 74,8) \\
36,6(16,7 ; 62,3)\end{array}$ \\
\hline $\begin{array}{l}\text { Zona geográfica, (\%) } \\
\text { Rural } \\
\text { Urbano }\end{array}$ & $\begin{array}{l}72,5(23,2 ; 95,8) \\
70,9(62,3 ; 78,2)\end{array}$ & $\begin{array}{l}27,5(4,16 ; 76,7) \\
29,1(21,7 ; 37,6)\end{array}$ \\
\hline $\begin{array}{l}\text { Nivel educacional, (\%) } \\
<8 \text { años } \\
\text { 8-12 años } \\
>12 \text { años }\end{array}$ & $\begin{array}{l}63,2(49,3 ; 75,0) \\
74,8(61,7 ; 84,6) \\
78,1(57,8 ; 90,2)\end{array}$ & $\begin{array}{l}36,8(24,9 ; 50,6) \\
25,1(15,3 ; 38,2) \\
21,8(9,74 ; 42,1)\end{array}$ \\
\hline $\begin{array}{l}\text { Características antropométricas } \\
\text { Peso }(\mathrm{Kg}) \\
\text { IMC }\left(\mathrm{kg} / \mathrm{m}^{2}\right) \\
\text { Perímetro cintura }(\mathrm{cm}) \\
\text { Obesidad central, }(\%)\end{array}$ & $\begin{array}{c}76,4(73,9 ; 78,8) \\
29,6(28,8 ; 30,5) \\
100,5(98,4 ; 102,6) \\
83,6(74,6 ; 89,7)\end{array}$ & $\begin{array}{l}68,3(64,9 ; 71,7) \\
27,1(26,0 ; 28,2) \\
92,2(88,0 ; 96,4) \\
16,4(10,2 ; 25,3)\end{array}$ \\
\hline $\begin{array}{l}\text { Estado nutricional, (\%) } \\
\text { Bajo peso } \\
\text { Normal } \\
\text { Sobrepeso } \\
\text { Obeso }\end{array}$ & $\begin{array}{l}54,3(37,1 ; 70,6) \\
74,1(61,0 ; 83,9) \\
87,4(74,3 ; 94,4) \\
30,4(10,3 ; 62,3)\end{array}$ & $\begin{array}{l}45,6(29,3 ; 62,8) \\
25,8(16,0 ; 38,9) \\
12,5(5,59 ; 25,6) \\
69,5(37,6 ; 89,6)\end{array}$ \\
\hline $\begin{array}{l}\text { Estilos de vida } \\
\text { Actividad física de transporte (min/día) } \\
\text { Actividad física moderada (min/día) } \\
\text { Actividad física Vigorosa (min/día) } \\
\text { Tiempo sedente (min/día) }\end{array}$ & $\begin{array}{c}71,3(38,8 ; 103,8) \\
221,6(154,6 ; 288,7) \\
201,8(96,1 ; 307,5) \\
193,9(161,4 ; 226\end{array}$ & $\begin{array}{c}60,6(28,2 ; 93,0) \\
149,0(84,6 ; 213,3) \\
185,1(-49,7 ; 420,1) \\
201,9(155,5 ; 248,3)\end{array}$ \\
\hline $\begin{array}{l}\text { Actividad física (\%) } \\
\text { Activo } \\
\text { Inactivo } \\
\text { Diabetes Mellitus tipo } 2 \text { (\%) } \\
\text { Hipertensión arterial (\%) } \\
\text { Síndrome metabólico (\%) }\end{array}$ & $\begin{array}{l}71,5(61,1 ; 80,0) \\
69,3(53,5 ; 81,3) \\
75,8(60,4 ; 86,6) \\
69,2(58,3 ; 78,2) \\
73,4(57,8 ; 84,8)\end{array}$ & $\begin{array}{l}28,4(19,9 ; 38,8) \\
30,6(18,6 ; 46,0) \\
24,1(13,3 ; 39,5) \\
30,8(21,7 ; 41,6) \\
26,5(15,1 ; 42,1)\end{array}$ \\
\hline $\begin{array}{l}\text { Bienestar personal, (\%) } \\
\text { Mala } \\
\text { Regular } \\
\text { Bien }\end{array}$ & $\begin{array}{l}42,3(16,2 ; 73,5) \\
55,3(38,4 ; 71,1) \\
80,5(71,3 ; 87,3)\end{array}$ & $\begin{array}{l}57,6(26,4 ; 83,7) \\
44,6(28,8 ; 61,5) \\
19,4(12,6 ; 28,6)\end{array}$ \\
\hline $\begin{array}{l}\text { Autopercepción de salud, (\%) } \\
\text { Mala } \\
\text { Regular } \\
\text { Buena }\end{array}$ & $\begin{array}{l}81,8(69,6 ; 89,8) \\
67,8(53,5 ; 79,4) \\
52,1(33,3 ; 70,3)\end{array}$ & $\begin{array}{l}18,1(10,1 ; 30,3) \\
32,1(20,5 ; 46,4) \\
47,8(29,6 ; 66,6)\end{array}$ \\
\hline
\end{tabular}

Datos presentados como promedio o prevalencia expandidas a población nacional y sus respectivos $95 \%$ IC. * Muestra encuestada corresponde a la población encuestada por la ENS y que fue incluida en este estudio. La muestra expandida corresponde a la extrapolación a población nacional realizada con los factores de expansión sugeridos por la ENS 2016-2017. 
también reportó un menor tiempo destinado a desarrollar actividad física en todos sus dominios, así como también un tiempo mayor destinado al desarrollo de actividades sedentes. Finalmente, los participantes con debilidad muscular señalaron peor percepción de bienestar personal.

La Figura 1 muestra la fuerza de prensión manual de los participantes según sexo y edad. Los niveles promedios de fuerza de prensión en personas mayores fueron de 35,3 kg [95\% IC: 30,7; $39,9$ ] y $19,7 \mathrm{~kg}$ [95\% IC: 17,$6 ; 21,7]$ para hombres y mujeres, respectivamente. Si bien la fuerza de prensión en todas las edades fue mayor en hombres que en mujeres, ambos sexos presentaron una disminución de sus niveles de fuerza muscular en la medida que incrementaron la edad. A los 60 años, los hombres evaluados alcanzaron en promedio $34,7 \mathrm{~kg}$ mientras que las mujeres de la misma edad presentaron 22,1 kg. Sin embargo, los niveles de fuerza muscular fueron $17 \%$ y $22 \%$ más bajos en personas de 90 años, llegando a $28,8 \mathrm{~kg}$ y $17,2 \mathrm{~kg}$ para hombres y mujeres, respectivamente (Figura 1).

La prevalencia de debilidad muscular, es decir tener niveles de fuerza $\leq 15 \mathrm{~kg}$ en mujeres $\mathrm{y} \leq 27 \mathrm{~kg}$ en hombres por edad se presenta en la Figura 2. La prevalencia de debilidad muscular en personas mayores fue mayor en hombres $(30,2 \%$ [95\% IC: 17,$3 ; 43,2])$ que en mujeres $(26,1 \%$ [95\% IC: $16,7 ; 35,5])$. Al analizar la prevalencia de debilidad muscular según edad, se evidenció que un 17,6\% y $17,5 \%$ de los hombres y mujeres de 60 años presentaron debilidad muscular. Esta prevalencia aumentó a $47,6 \%$ y $34,3 \%$ a los 80 años, llegando a $79,3 \%$ y $56,3 \%$ en hombres y mujeres centenarias, respectivamente.

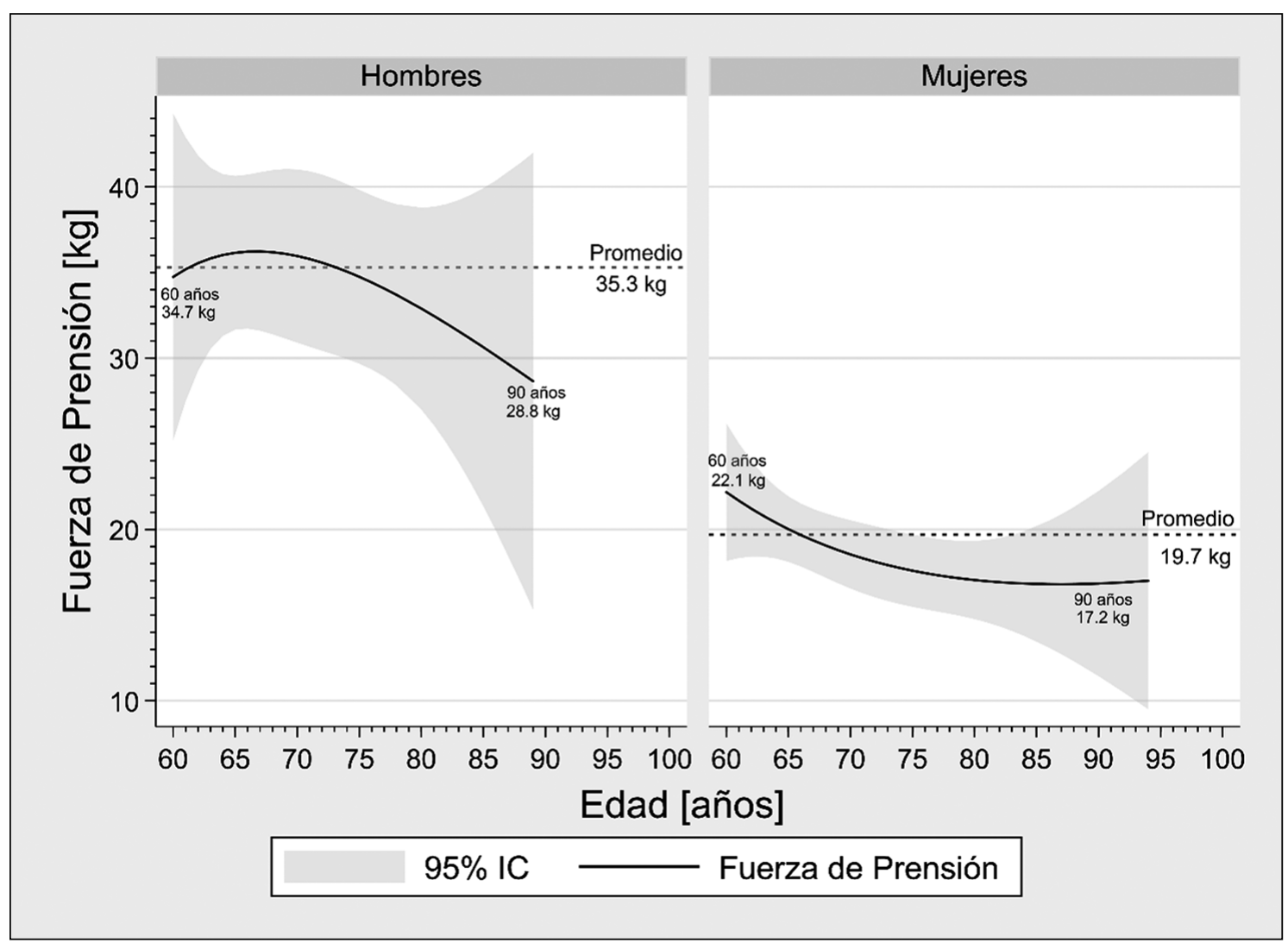

Figura 1. Fuerza de prensión manual según sexo y edad en personas mayores chilenas. Datos presentados como promedios de fuerza de prensión en $\mathrm{Kg}$ con sus respectivos $95 \%$ IC y ponderados para población nacional. El promedio reportado corresponde a la fuerza de prensión promedio para hombres y mujeres en Chile. El área gris representa los $95 \%$ IC de las estimaciones de fuerza de prensión según la edad. 


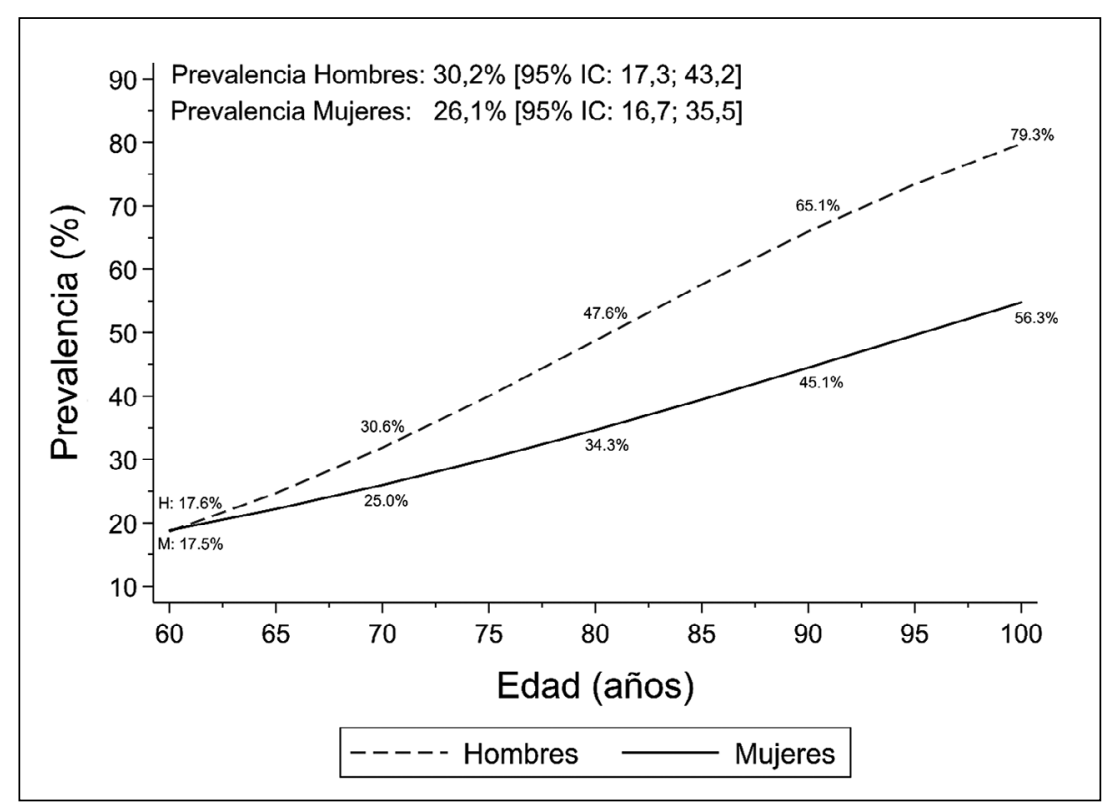

Figura 2. Prevalencia de debilidad muscular según sexo y edad en personas mayores chilenas. Datos presentados como prevalencias ponderadas a población nacional. Se consideró con debilidad muscular a las mujeres que obtuvieron $\leq 15 \mathrm{y} \leq 27 \mathrm{~kg}$ en hombres en la prueba de fuerza de prensión manual.

\section{Discusión}

El principal resultado de este estudio revela que la fuerza de prensión manual disminuye con la edad en ambos géneros a partir de los 60 años; no obstante, esta disminución fue más acentuada en hombres que en mujeres. Si bien dicha prevalencia fue similar a los 60 años, a mayor edad las diferencias fueron más acentuadas $(79,3 \%$ versus 56,3\% en hombres y mujeres de 100 años, respectivamente).

Nuestros resultados concuerdan con los reportados por estudios previos los cuáles, plantean que la fuerza muscular declina más rápido en hombres, al menos después de los 64 años ${ }^{25,26}$. Por otra parte, Doods y cols. reportaron un descenso de un $23 \%$ y $27 \%$ en la fuerza de prensión manual en hombres y mujeres de 80 años, valores por debajo de los encontrados en esta investigación ${ }^{26}$. Estas diferencias podrían atribuirse a las diferentes metodologías de evaluación utilizadas y también a las diferencias entre la población estudiada ${ }^{27,28}$. A nivel nacional, Mancilla y cols. reportaron una importante disminución de la fuerza de prensión manual en personas mayores, siendo sus hallazgos coincidentes con los entregados por esta investigación ${ }^{14}$.

Se ha postulado que la pérdida de masa y fuerza muscular comienza aproximadamente a los 30 años, donde se pierde masa debido a que las proteínas contráctiles de las fibras musculares disminuyen, siendo a veces reemplazadas por tejido conjuntivo y adiposo ${ }^{29}$. Sumado a lo anterior, al interior de las fibras musculares se ha evidenciado una reducción de la expresión de los canales de $\mathrm{Ca}^{2+}$ tipo L, reduciendo de este modo el peak de $\mathrm{Ca}^{2+}$ citosólico. Entre las consecuencias de este proceso, se identifica la disminución de la capacidad de generar fuerza en cada contracción muscular ${ }^{7,30}$.

Similarmente, se ha reportado que algunos eventos asociados al envejecimiento como cambios en la sensibilidad a la insulina, menopausia, caquexia y obesidad sarcopénica provocan cambios hormonales, entre ellos la reducción de andrógenos (testosterona), estrógenos y hormonas del crecimiento (IGF-1- factor de crecimiento similar a la insulina), así también aumento de citoquinas antiinflamatorias y proinflamatorias como las interleuquinas IL-1 (interleuquina-1), IL-6 (interleuquina -6), IL-15y TNF- $\alpha$ (factor de necrosis tumoral- $\alpha$ ), las cuales pueden modular la homeostasis entre la síntesis de proteínas y la degradación muscular ${ }^{7}$. Se ha observado que un incremento en la producción de citoquinas proinflamatorias, principalmente IL-6, o modificaciones de la sensibilidad a citoquinas por parte de las células musculares envejecidas podría 
contribuir a cambios en la masa y función muscular, generando debilidad y pérdida de fuerza, lo que funcionalmente se traduce en mayor riesgo de caídas, pérdida funcional y fragilidad ${ }^{7,31}$. De igual modo el estudio Framingham reportó que mayores niveles o producción de las citocinas catabólicas como TNF- $\alpha$ e IL- 6 se asocian con una mayor mortalidad en adultos mayores que viven en la comunidad ${ }^{32}$.

Investigaciones previas posicionan a la prueba de prensión manual como una evaluación útil y básica para la determinación de la función musculoesquelética, así como también de debilidad, sarcopenia, fragilidad, discapacidad y mortalidad en personas mayores ${ }^{11,33,34}$. Dichos estudios evidencian, además, que la fuerza de prensión disminuye con la edad, coincidiendo con los hallazgos encontrados en esta investigación. Bohannon y cols. identificaron que la fuerza de prensión manual permite no solo identificar la debilidad muscular de extremidad superior, sino que también proporciona un indicador de la fuerza general, ya que refleja la fuerza de las extremidades inferiores ${ }^{35}$. Esto le da un papel importante en la evaluación de la funcionalidad de las personas mayores ${ }^{35}$. En este contexto, este tipo de medición de fácil aplicación, alta precisión y bajo costo podría ser fácilmente incorporada en el screening de personas con mayor riesgo de enfermedades crónicas, fragilidad y mortalidad prematura.

Si bien este estudio presenta fortalezas como la medición objetiva de fuerza de prensión mediante protocolos estandarizados de medición, no está exento de limitaciones. Por ejemplo, si bien los resultados de esta investigación coinciden con los reportes internacionales y nacionales ${ }^{14,17}$ solo se incluyó una submuestra de personas mayores de la región metropolitana lo que impide la generalización de los hallazgos a toda la población mayor chilena. Por ende, los niveles de fuerza de la población que vive en otras regiones del país podrían ser diferentes a los encontrados en esta submuestra. Estudios futuros deberían incluir esta medición a nivel nacional y no solo restringirla a personas mayores de una región particular.

\section{Conclusión}

La fuerza de prensión manual disminuyó con la edad en ambos sexos a partir de los 60 años, no obstante, esta disminución fue más acentuada en hombres que en mujeres. Estos hallazgos podrían tener importantes implicaciones para la salud pública chilena, ya que la fuerza de prensión manual, en comparación con otras medidas físicas, es un instrumento fácil de medir, de bajo costo y altamente reproducible en la práctica clínica, por lo que podría incluirse como test de tamizaje para conocer la condición músculo-esquelética de las personas mayores.

\section{Referencias}

1. Thomas E, Battaglia G, Patti A, Brusa J, Leonardi V, Palma A, et al. Physical activity programs for balance and fall prevention in elderly: A systematic review. Medicine 2019; 98 (27).

2. Seidler RD, Alberts JL, Stelmach GE. Changes in multi-joint performance with age. Motor control 2002; 6 (1): 19-31.

3. Ricci NA, Aratani MC, Doná F, Macedo C, Caovilla HH, Ganança FF. Revisão sistemática sobre os efeitos da reabilitação vestibular em adultos de meia-idade e idosos. Braz J Phys Ther 2010; 14: 361-71.

4. Brook MS, Wilkinson DJ, Phillips BE, Pérez-Schindler J, Philp A, Smith K, et al. Skeletal muscle homeostasis and plasticity in youth and ageing: impact of nutrition and exercise. Acta Physiologica 2016; 216 (1): 15-41.

5. Virani SS, Wang D, Woodard LD, Chitwood SS, Landrum CR, Zieve FJ, et al. Non-high-density lipoprotein cholesterol reporting and goal attainment in primary care. J Clin Lipidol 2012; 6 (6): 545-52.

6. Fielding RA, Vellas B, Evans WJ, Bhasin S, Morley JE, Newman AB, et al. Sarcopenia: an undiagnosed condition in older adults. Current consensus definition: prevalence, etiology, and consequences. International working group on sarcopenia. J Am Med Dir Assoc 2011; 12 (4): 249-56.

7. Argilés JM, Busquets S, López-Soriano FJ, Figueras M. Fisiología de la sarcopenia: Similitudes y diferencias con la caquexia neoplásica. Nutr Hosp 2006; 21: 38-45.

8. Serratrice G, Roux H, Aquaron R. Proximal muscular weakness in elderly subjects: report of 12 cases. J Neurol Sci 1968; 7 (2): 275-99.

9. Clark BC, Manini TM. What is dynapenia? Nutrition 2012; 28 (5): 495-503.

10. Roberts HC, Denison HJ, Martin HJ, Patel HP, Syddall $\mathrm{H}$, Cooper $\mathrm{C}$, et al. A review of the measurement of grip strength in clinical and epidemiological studies: towards a standardised approach. Age and ageing 2011; 40 (4): 423-9. 
11. Celis-Morales CA, Welsh P, Lyall DM, Steell L, Petermann F, Anderson J, et al. Associations of grip strength with cardiovascular, respiratory, and cancer outcomes and all cause mortality: prospective cohort study of half a million UK Biobank participants. BMJ 2018; 361: 1651.

12. Leong DP, Teo KK, Rangarajan S, Lopez-Jaramillo P, Avezum Jr A, Orlandini A, et al. Prognostic value of grip strength: findings from the Prospective Urban Rural Epidemiology (PURE) study. Lancet 2015; 386 (9990): 266-73.

13. Ho FK, Celis-Morales CA, Petermann-Rocha F, Sillars A, Welsh P, Welsh C, et al. The association of grip strength with health outcomes does not differ if grip strength is used in absolute or relative terms: a prospective cohort study. Age and ageing 2019; 48 (5): 684-91.

14. Mancilla E, Ramos S, Morales P. Fuerza de prensión manual según edad, género y condición funcional en adultos mayores Chilenos entre 60 y 91 años. Rev Med Chile 2016; 144 (5): 598-603.

15. Gómez-Campos R, Andruske CL, Arruda Md, Sulla-Torres J, Pacheco-Carrillo J, Urra-Albornoz C, et al. Normative data for handgrip strength in children and adolescents in the Maule Region, Chile: Evaluation based on chronological and biological age. PloS one 2018; 13 (8): e0201033.

16. Romero-Dapueto C, Mahn J, Cavada G, Daza R, Ulloa V, Antúnez M. Estandarización de la fuerza de prensión manual en adultos chilenos sanos mayores de 20 años. Rev Med Chile 2019; 147 (6): 741-50.

17. Lera L, Albala C, Leyton B, Márquez C, Angel B, Saguez $\mathrm{R}$, et al. Reference values of hand-grip dynamometry and the relationship between low strength and mortality in older Chileans. Clin Interv Aging 2018; 13: 317.

18. (MINSAL) Ministerio de Salud. Departamento de Epidemiología. Encuesta Nacional de Salud 2016-2017.

19. Cruz-Jentoft AJ, Bahat $G$, Bauer J, Boirie $Y$, Bruyère O, Cederholm T, et al. Sarcopenia: revised European consensus on definition and diagnosis. Age and Ageing 2019; 48 (1): 16-31.

20. OPS. Parte 1: Módulos de Valoración clínica. Módulo 5: Valoración Nutricional del Adulto Mayor. editor. 2003.

21. (MINSAL) Ministerio de Salud. Encuesta Nacional de Salud 2009-2010. Chile: Ministerio de Salud; 2010.

22. Bull FC, Maslin TS, Armstrong T. Global physical activity questionnaire (GPAQ): nine country reliability and validity study. Journal of Physical Activity and health. 2009; 6 (6): 790-804.

23. Hoos T, Espinoza N, Marshall S, Arredondo EM. Validity of the global physical activity questionnaire (GPAQ) in adult Latinas. Journal of Physical Activity and Health. 2012; 9 (5): 698-705.

24. WHO. Organización Mundial de la Salud. Global Physical Activity Questionnaire: GPAQ version 2.0. 2009.

25. Hirsch CH, Fried LP, Harris T, Fitzpatrick A, Enright $P$, Schulz R, et al. Correlates of performance-based measures of muscle function in the elderly: the Cardiovascular Health Study. J Gerontol A Biol Sci Med Sci 1997; 52 (4): M192-M200.

26. Dodds RM, Syddall HE, Cooper R, Benzeval M, Deary IJ, Dennison EM, et al. Grip strength across the life course: normative data from twelve British studies. PloS one 2014 ; 9 (12).

27. Curcio CL, Gómez JF. Fuerza de agarre de los adultos mayores de los centros día del municipio de Manizales. Rev Asoc Colomb Gerontol Geriatr 2005; 19 (4): 849-58.

28. Giraldo J, Giraldo D, Suárez C, Curcio C. Fuerza de agarre en hombres ancianos ambulatorios. Rev Asoc Colomb Gerontol Geriatr 2003; 17 (1): 455-61.

29. Janssen I, Heymsfield SB, Ross R. Low relative skeletal muscle mass (sarcopenia) in older persons is associated with functional impairment and physical disability. J Am Geriatr Soc 2002; 50 (5): 889-96.

30. Delbono O. Molecular mechanisms and therapeutics of the deficit in specific force in ageing skeletal muscle. Biogerontology 2002; 3 (5): 265-70.

31. Feldman HA, Longcope C, Derby CA, Johannes CB, Araujo AB, Coviello AD, et al. Age trends in the level of serum testosterone and other hormones in middle-aged men: longitudinal results from the Massachusetts male aging study. J Clin Endocrinol Metab 2002; 87 (2): 589 98.

32. Roubenoff R, Parise H, Payette HA, Abad LW, D'Agostino R, Jacques PF, et al. Cytokines, insulin-like growth factor 1 , sarcopenia, and mortality in very old community-dwelling men and women: the Framingham Heart Study. Am J Med 2003; 115 (6): 429-35.

33. Celis-Morales CA, Lyall DM, Anderson J, Iliodromiti S, Fan Y, Ntuk UE, et al. The association between physical activity and risk of mortality is modulated by grip strength and cardiorespiratory fitness: evidence from 498135 UK-Biobank participants. Eur Heart J 2017; 38 (2): 116-22.

34. Amaral CA, Amaral TLM, Monteiro GTR, Vasconcellos MTL, Portela MC. Hand grip strength: Reference values for adults and elderly people of Rio Branco, Acre, Brazil. PloS One 2019; 14 (1): e0211452.

35. Bohannon RW. Are hand-grip and knee extension strength reflective of a common construct? Percept Mot Skills 2012; 114 (2): 514-8. 